\title{
Biomarkers of Fibroproliferative Healing in Fibrosing Idiopathic Interstitial Pneumonias
}

\author{
Martina Vasakova ${ }^{*}, 1$, Martina Sterclova ${ }^{1}$, Eliska Stranska ${ }^{2}$, Petra Mandakova ${ }^{2}$, \\ Jelena Skibova ${ }^{3}$ and Radoslav Matej ${ }^{4}$
}

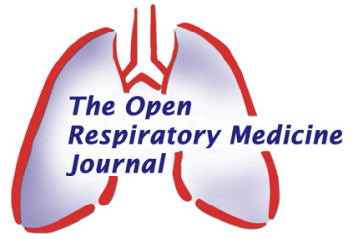

\author{
${ }^{I}$ Department of Respiratory Medicine, Thomayer Hospital, Videnska 800, 14059 Prague 4, Czech Republic \\ ${ }^{2}$ Department of Pathology and Molecular Medicine, Charles University in Prague, $2^{\text {nd }}$ Medical Faculty, V Uvalu 84, \\ 15006 Prague 5, Czech Republic \\ ${ }^{3}$ Medical Statistics Unit, Institute for Clinical and Experimental Medicine, Videnska 1958/9, 14021 Prague 4, Czech \\ Republic \\ ${ }^{4}$ Department of Pathology and Molecular Medicine, Thomayer Hospital, Videnska 800, 14059 Prague 4, Czech \\ Republic
}

\begin{abstract}
Aims: The main feature of fibrosing idiopathic interstitial pneumonias (fIIPs) is the fibroproliferative potential of underlying pathogenetic process. We hypothesize that the concentration of potential markers of fibroproliferative healing, PAR-2, TGF- $\beta 1$, TNF- $\alpha$ and IL-4R $\alpha$ in bronchoalveolar lavage fluid (BALF) differ in patients with fIIPs compared to controls (C).
\end{abstract}

Patients and Methods: 10 patients with fIIPs and 9 controls (C) were included to the study.

Concentrations of CD124 (IL4R $\alpha$ ), PAR-2, TGF- $\beta 1$ and TNF- $\alpha$ in BALF were determined using the ELISA method.

Results: We observed higher concentrations of IL4R $\alpha$ (fIIPS $1499.4 \mathrm{pg} / \mathrm{ml} v s$ C $255.5 \mathrm{pg} / \mathrm{ml} ; \mathrm{p}<0.05$ ), PAR-2 (fIIPS $1807.9 \mathrm{pg} / \mathrm{ml}$ vs C $421.0 \mathrm{pg} / \mathrm{ml} ; \mathrm{p}<0.05$ ) and TGF- $\beta 1$ (fIIPS $283.0 \mathrm{pg} / \mathrm{ml} v s \mathrm{C} 197.1 \mathrm{pg} / \mathrm{ml} ; \mathrm{p}<0.01$ ) in BALF in fIIPs versus $\mathrm{C}$. The values of TNF- $\alpha$ in BALF did not differ significantly in fIIPs compared to controls. The ratios also showed differences in fIIPS and C: IL4R $\alpha /$ TGF- $\beta 1$ (fIIPS $6.19 v s \mathrm{C} 0.68 ; \mathrm{p}=0.0143$ ); TNF- $\alpha /$ IL4R $\alpha$ (fIIPS $0.84 v s \mathrm{C} 7.93 ; \mathrm{p}=$ 0.043 ); TGF- $\beta 1 /$ TNF- $\alpha$ (fIIPS 0.21 vs $\mathrm{C} 0.16 ; \mathrm{p}=0.0179$ ) and protein/PAR-2 (fIIPS 0.06 vs $\mathrm{C} 0.28 ; \mathrm{p}=0.0033$ ).

Conclusions: We found that PAR-2, TGF- $\beta 1$ and IL-4R $\alpha$ are significantly up-regulated in the BALF of fIIPs compared to controls, therefore we suppose they could become biomarkers of fibroproliferative healing.

Keywords: Idiopathic interstitial pneumonias, fibroproliferative healing, biomarkers, bronchoalveolar lavage fluid, PAR-2, TGF-beta, IL4R.

\section{INTRODUCTION}

Fibrosing idiopathic interstitial pneumonias (fIIPs), comprising mainly idiopathic pulmonary fibrosis (IPF) and fibrotic idiopathic nonspecific interstitial pneumonia (iNSIP), are processes with unknown etiology and a grim prognosis. The main feature influencing the poor clinical outcome of fIIPs is the fibroproliferative potential of the underlying pathogenetic process in the lung, which is mediated by the $\mathrm{T}_{\mathrm{H}} 2$ immune response. We hypothesize that the cytokine milieu in bronchoalveolar lavage fluid (BALF) in fIIPs is different from controls and directly enhances fibroproliferative healing in the lung. We also propose that the values of cytokines and receptors involved in cytokine signaling in BALF could be useful biomarkers of fibroproliferative healing in interstitial lung diseases (ILDs).

*Address correspondence to this author at the Department of Respiratory Medicine, Thomayer Hospital Prague, Videnska 800, 14059 Prague 4, Czech Republic; Tel: + 42026108 2372; Fax: + 42026108 2206;

E-mail: martina.vasakova@ftn.cz
We have concentrated primarily on the cytokines and receptors which are potentially involved in fibroproliferative healing. After many studies, it is now well recognized that a number of key growth factors are responsible for driving the process of fibrogenesis. For example, transforming growth factor-beta 1 (TGF- $\beta 1$ ), interleukin-1 beta (IL-1 $\beta$ ), and tumor necrosis factor-alpha (TNF- $\alpha$ ) are able to induce the characteristic motility, proliferation and extracellular matrix (ECM) synthesis observed in mesenchymal cells with a myofibroblast-like phenotype from fibroblastic foci [1-5]. One of the key receptors in the initiation of fibroproliferative healing is the proteinase-activated receptor-2 (PAR-2), which interconnects activation of the coagulation cascade in alveolar injury with consequent fibroproliferative healing. In a study by Wygrecka et al., PAR-2 was strongly upregulated in IPF lungs and was attributed to alveolar type II cells and fibroblasts/myofibroblasts. TGF- $\beta 1$, a key profibrotic cytokine, considerably enhances PAR-2 expression in human lung fibroblasts. FVIIa stimulates proliferation of human lung fibroblasts and ECM production 
in a PAR-2-dependent manner, but did not initiate differentiation of fibroblasts into myofibroblasts [6,7]. TGF$\beta 1$ increases fibroblast proliferation, stimulates synthesis and deposition of connective tissue, and inhibits connective tissue breakdown. TGF- $\beta 1$ acts through a promoter of the type 1 collagen gene causing increased collagen synthesis. In addition, TGF- $\beta 1$ induces epithelial-mesenchymal transition (EMT) in alveolar epithelial cells (AECs), both in vitro and in vivo [8-12].

TNF- $\alpha$ plays an important pro-inflammatory role and alveolar macrophages are the main source of this cytokine [12]. It appears that in IPF TNF- $\alpha$ probably plays a pathogenic role in the early phase of the disease, i.e. during alvelolar epithelial injury, and can be regulated by antioxidants $(\mathrm{Cu})$. Also, alternatively activated macrophages, after lipopolysaccharide (LPS)-induced PAR-2 activation, have in vitro, been shown to suppress mRNA expression of pro-inflammatory cytokines including TNF- $\alpha$ [13].

The key cell inducing fibroproliferative healing in fIIPs is the alveolar macrophage, which can be alternatively activated by interleukin (IL)-13 [14,15]. IL-13 in addition to IL-4 is a key inducer of several type- 2 cytokine-dependent pathologies. It regulates inflammation, mucus production, tissue remodeling, and fibrosis. IL-13 signaling is mediated by the type- 2 IL-4 receptor, which consists of IL-4R $\alpha$ and IL-13R $\alpha 1$ chains [16]. Pulmonary fibroblast lines cultured from patients with the most severe form of IIP, i.e. usual interstitial pneumonia, exhibited the greatest gene and protein expression of IL-4R $\alpha$, IL-13R $\alpha 1$, and IL-13R $\alpha 2$ compared with primary pulmonary fibroblast lines grown from other IIP surgical lung biopsies (SLBs) and normal SLBs. The fibroblast lines from IIP also had a greater response (increased inhibition) to chimeric proteins comprised of human IL-13 and truncated versions of the Pseudomonas exotoxin (IL-13-PE), compared to normal fibroblasts; the response was probably dependent on their expression of IL-4R $\alpha$ and IL-13R $\alpha 2$ [17].

\section{AIM}

According the results of previous studies and to our preliminary studies in ILDs, we have targeted this investigation of potential biomarkers in BALF in fIIPS (IPF, fibrotic NSIP) [18,19]. We hypothesize that the concentration of potential markers of fibroproliferative healing, i.e. PAR-2, TGF- $\beta 1$, TNF- $\alpha$ and IL-4R $\alpha$ differ in patients with fIIPs compared to controls and as such, could be useful biomarkers of fibroproliferative healing in interstitial lung diseases.

\section{PATIENTS AND METHODS}

\section{Patients}

Ten patients with fIIPs ( 4 IPF and 6 fibrotic idiopathic NSIP) were included in the study. The patients did not receive any treatment for fIIPS at the time of entering the study and having lower mentioned investigations. The demographic data are presented in Table 1. The diagnosis in fIIPs was based on the clinical picture, HRCT, and transbronchial ( 7 patients) or surgical lung biopsy (3 patients). Nine patients with pneumonia in regression with suspicion of interstitial lung disease, which was not consequently proven by HRCT, served as the control group
(C). All patients signed an informed consent before entering the study and the study protocol was approved by the Central Ethics Committee of Thomayer Hospital and the Institute for Clinical and Experimental Medicine. Additionally, all data were analyzed with respect for patient privacy.

Table 1. Basic Characteristics of fIIPS and C Groups

\begin{tabular}{|c|c|c|c|}
\hline & $\begin{array}{c}\text { No/Gender } \\
(\mathbf{M} / \mathbf{F})\end{array}$ & Age & $\begin{array}{c}\text { Smoking } \\
\text { (Nonsmokers/Exsmokers) }\end{array}$ \\
\hline \hline $\mathrm{C}$ & & & $6 / 3$ \\
\hline median & $9(6 / 3)$ & $\mathbf{6 6}$ & \\
\hline min & & 30 & \\
\hline max & & 73 & \\
\hline mean & & 60.33 & \\
\hline $\mathrm{SD}$ & & 13.07 & \\
\hline & & & \\
\hline fIIPs & & & \\
\hline $\mathrm{n}$ & $10(3 / 7)$ & & \\
\hline median & & $\mathbf{6 8}$ & \\
\hline min & & 48 & \\
\hline max & & 79 & \\
\hline mean & & 65.40 & \\
\hline SD & & 9.36 & \\
\hline Statistics & NS (F test) & NS (M-W test) & \\
\hline
\end{tabular}

$\mathrm{M} / \mathrm{F}$, male/female; C, controls; fIIPs, fibrosing idiopathic interstitial pneumonias; SD, standard deviation; M-W test, Mann-Whitney test, Fisher's exact test.

\section{Methods}

Bronchoalveolar lavage (BAL) was performed in both fIIPs groups and the $\mathrm{C}$ group during fiberoptic bronchoscopy, which was done under local anesthesia. Five fractions of $50 \mathrm{ml}$ of lukewarm saline were instilled into the segmental part of the middle lobe where the bronchoscope was wedged. The fluid was retrieved using syringe suction and mixed in a container before being divided for further investigation. The sample was determined to be valid if the recovery exceeded $20 \mathrm{ml}$ per fraction and a significant admixture of polymorphic bronchial epithelial cells was not found. At least $25 \mathrm{ml}$ of BALF was sent for further investigation of cytokine values. Concentrations of CD124 (IL-4R $\alpha$ ), PAR-2, TGF- $\beta 1$ and TNF- $\alpha$ in BALF were determined using the ELISA method, using kits from Uscn Life Science Inc. Reactions took place in microtiter plate wells which had been pre-coated with monoclonal antibody (MAb) specific for the examined analyte (CD124, PAR-2, TGF- $\beta 1$ and TNF- $\alpha$ ). Labeled polyclonal antibody binds to the MAb-analyte complex. After reacting with the substrate solution the process is terminated. The colored product was measured with a vertical colorimeter Biotek EL800. The concentration of analyte in samples was determined using a standard curve. To avoid disturbances and potential errors related to different BALF sample amounts and thus different 
Table 2. Concentrations of Cytokines and Receptors in BALF in fIIPs and C

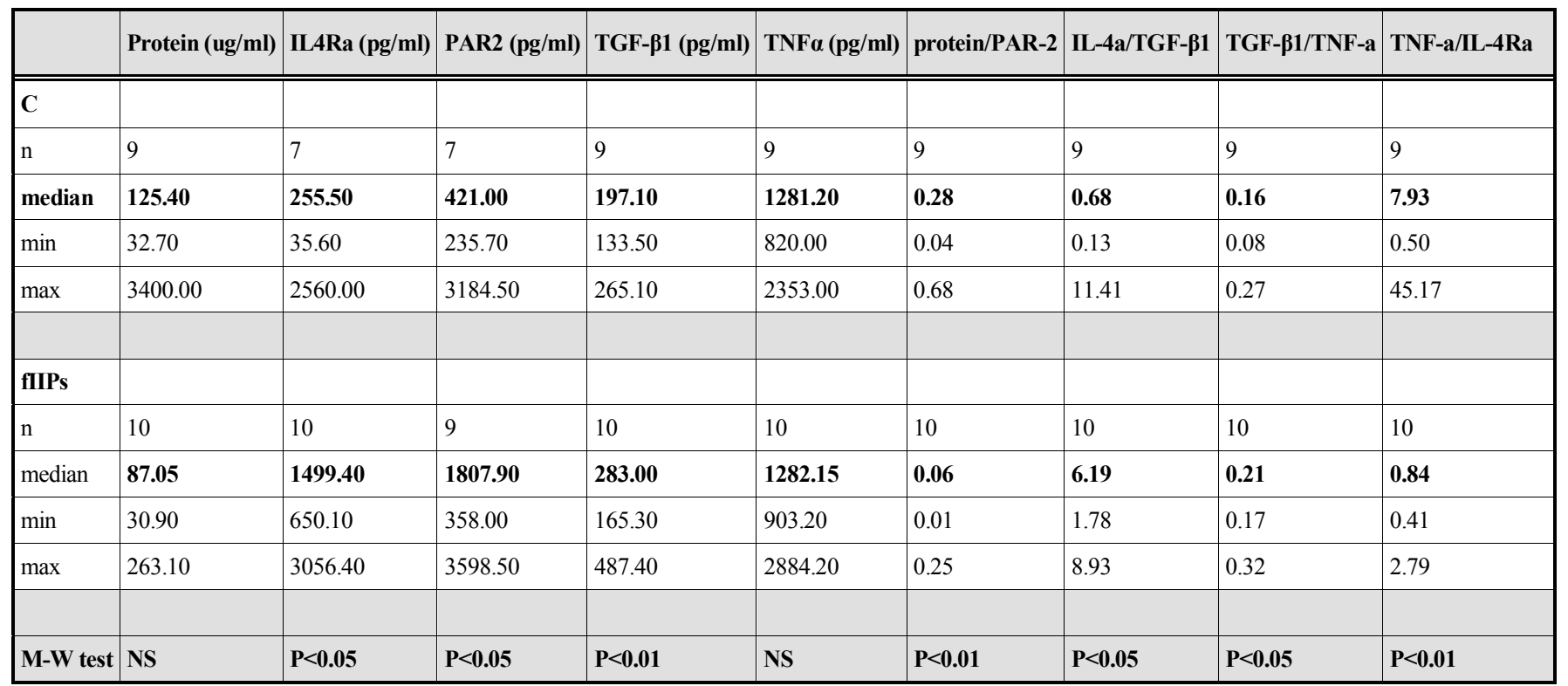

C, controls; fIIPs, fibrosing idiopathic interstitial pneumonias; SD, standard deviation; M-W test, Mann-Whitney test.

concentration of cytokines and receptors, we also measured total protein values in the BALF and present the cytokines and receptors values as ratios. The extreme values were excluded from statistical evaluation (Table 2).

\section{Statistical Methods}

Descriptive statistics were calculated, i.e. mean and standard deviation, median, minimal and maximal values. For discrete variables (gender, smoking status) the frequencies are presented. The Fisher's exact test was used for statistical evaluation of discrete variables and the MannWhitney test for continuous variables. The median values of the concentrations of cytokines and receptors in BALF were used for further statistical testing. The Mann-Whitney test was applied for comparison of cytokines and receptors values in BALF of fIIPS and $\mathrm{C}$ groups. Statistical analysis was performed using MedCalc statistical software. A p value less than 0.05 was considered significant.

\section{RESULTS}

We observed significantly higher concentrations of IL4R $\alpha$ (fIIPS $1499.4 \mathrm{pg} / \mathrm{ml} v s$ C $255.5 \mathrm{pg} / \mathrm{ml} ; \mathrm{p}<0.05$ ), PAR-2 (fIIPS $1807.9 \mathrm{pg} / \mathrm{ml}$ vs C $421.0 \mathrm{pg} / \mathrm{ml}$; $\mathrm{p}<0.05$ ) and TGF- $\beta 1$ (fIIPS $283.0 \mathrm{pg} / \mathrm{ml}$ vs C $197.1 \mathrm{pg} / \mathrm{ml} ; \mathrm{p}<0.01$ ) in BALF in fIIPs versus C, see Fig. (1). The values of TNF- $\alpha$ in BALF did not differ significantly in fIIPs compared to controls (Table 2). The ratios also showed statistically significant differences in concentrations of cytokines and receptors in fIIPS and C: IL4R $\alpha /$ TGF- $\beta 1$ (fIIPS 6.19 vs $\mathrm{C}$ $0.68 ; \mathrm{p}=0.0143$ ); TNF- $\alpha /$ IL4R $\alpha$ (fIIPS 0.84 vs C 7.93; $\mathrm{p}=$ 0.043 ); TGF- $\beta 1 /$ TNF- $\alpha$ (fIIPS 0.21 vs C $0.16 ; \mathrm{p}=0.0179$ ) and protein/PAR-2 (fIIPS 0.06 vs C $0.28 ; \mathrm{p}=0.0033$ ), see Fig. (2).

\section{DISCUSSION}

Fibrosing idiopathic interstitial pneumonias are serious diseases with a grim prognosis, however when they are diagnosed early they can be treated with novel anti-fibrotic drugs. Thus it is essential to make the diagnosis of fIIPs as early as possible. In the early stages, fIIPs are often described by radiologists as nonspecific or probably postinflammatory changes seen on high resolution computed tomography (HRCT) scans. In that stage of the disease, the BAL sometimes offers the only chance to obtain further information about the nature of interstitial lung disease, since not all the patients are able and/or want to undergo a surgical lung biopsy (SLB). Thus there is urgent need for reliable biomarkers which could support a diagnosis of early fIIPs and offer patients anti-fibrotic treatment in the early stages of the disease. The markers which have been introduced in fibrosing ILDs to date, i.e. KL-6 and surfactant protein-A (SP-A), are fundamentally markers of disease progression and acute exacerbation rather than early markers of the disease [20,21].

In our study we tried to identify potential biomarkers of fibroproduction from BALF of patients with known fIIPs, which could be used to identify patients with fIIPs even in early stages, absent acute exacerbation. We focused on molecules previously known to have an important role in fibroproliferative cytokine and receptor network.

We observed significantly higher concentrations of IL4R $\alpha$ in the BALF of patients with fIIPs compared to controls. Our findings support the results of previous studies by Mentink- Kane (2011), where IL-13 signaling, which is important for $\mathrm{T}_{\mathrm{H}} 2$ commitment of alveolar macrophages, is realized by the type-2 IL-4 receptor [16]. Also pulmonary fibroblasts in IPF exhibited the greatest gene and protein expression of IL-4R $\alpha$, IL-13R $\alpha 1$, and IL-13R $\alpha 2$ compared with primary pulmonary fibroblast lines grown from other IIPs and normal SLBs [17].

With regard to PAR-2, its concentration in BALF was also significantly elevated in fIIPs compared to controls. PAR-2 is a quite novel molecular marker of fibroproduction which connects the cogulation cascade axis during alveolar damage with consequent fibroproliferative healing $[6,7,13$, 


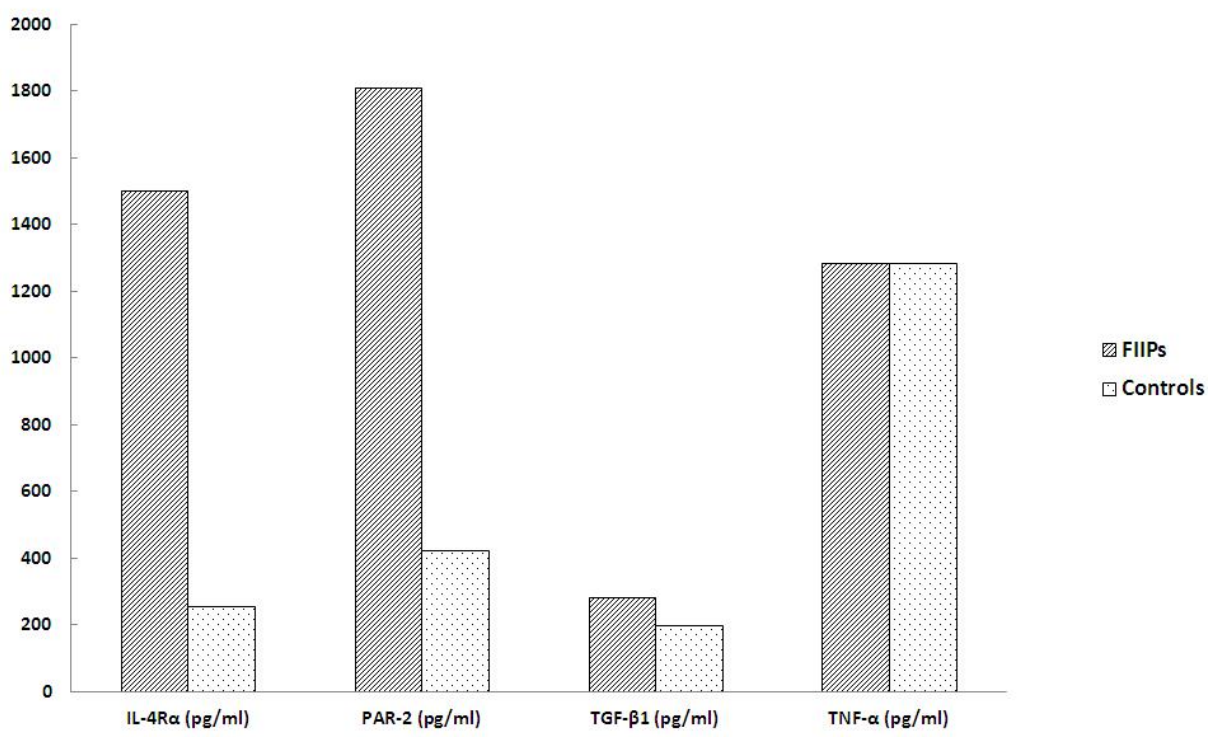

Fig. (1). Median values of TGF-b, TNF-a, IL-4Ra and PAR-2 in patients with fIIPs and controls.

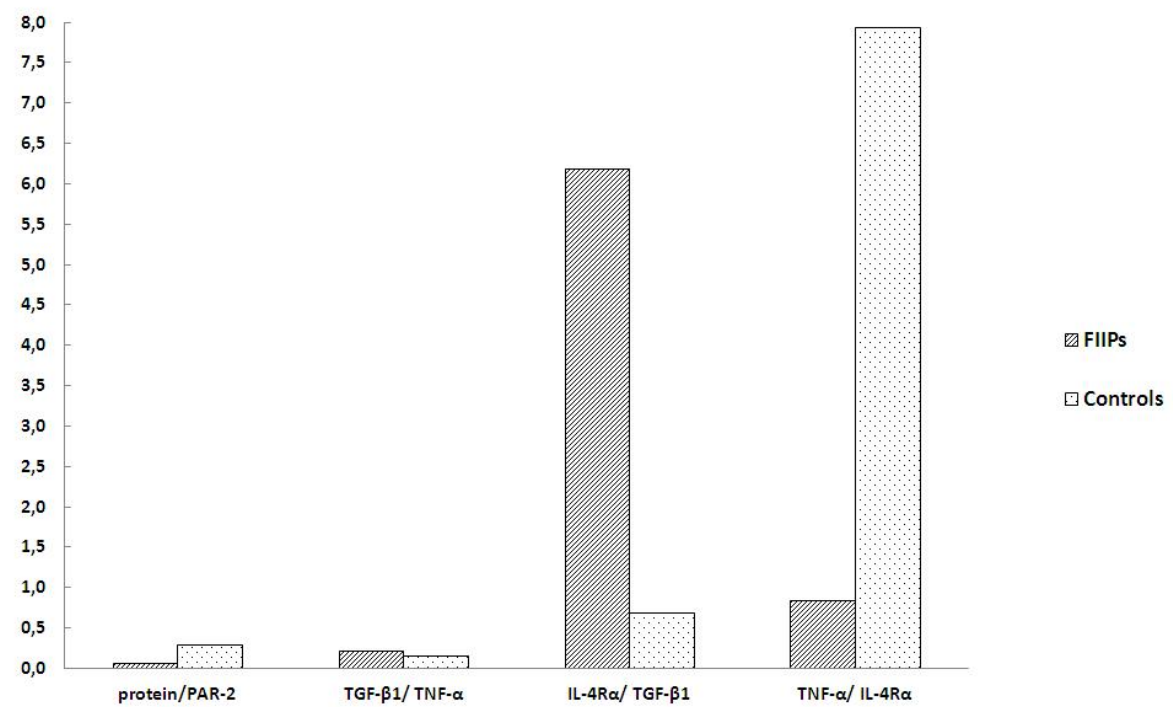

Fig. (2). Median values of protein/PAR-2, IL-4 $\alpha /$ TGF- $\beta 1$, TGF- $\beta 1 / T N F-\alpha$ and TNF- $\alpha /$ IL-4R $\alpha$ ratios in patients with fIIPs and controls

22,23]. Our findings supports the role of PAR-2 in fibroproliferative healing and suggests that PARs-2 could be a useful biomarker of fIIPs. Also the protein/PAR-2 ratio showed a significant prevalence of PAR-2 in the BALF of fIIPs compared to controls. TGF- $\beta 1$ is considered the dominant cytokine in fibroproliferative healing in fIIPs and our study confirmed that values of TGF- $\beta 1$ in the BALF of fIIPs were increased compared to controls [2-5,8-11,24].

TNF- $\alpha$, which has been reported as having a role in the initial phases of fIIPs, did not show any differences between fIIPs and controls [12,25]. Therefore, in our opinion, TNF- $\alpha$ is not a good biomarker candidate for fIIPs, with the exception of its use in ratios (see below).

To avoid the effect of different BALF dilutions, ratios of cytokines and receptors can be used as complex biomarkers.
We observed the following: IL4R/TGF- $\beta 1$ and TGF- $\beta$ $1 /$ TNF- $\alpha$ showed significantly higher values in fIIPs compared to $\mathrm{C}$, while TNF- $\alpha /$ IL4R $\alpha$ showed significantly lower values in fIIPS compared to $C$; as such, these ratios could also be considered good biomarkers of fibrotic interstitial lung diseases.

Further studies are needed to support our findings using larger groups of fIIPs patients and other ILDs and to establish their potential practical applications. This will be one of the main task of our next study.

\section{CONCLUSIONS}

Our results support the hypothesis regarding the role of the $\mathrm{T}_{\mathrm{H}} 2$ type immune response in the pathogenesis of fibrotic fIIPs. The up-regulation of IL- $4 \mathrm{R} \alpha$ might be in response to 
enhanced IL-4 and IL-13 production, which can lead to the profibrotic milieu in lung tissue. Increased values of TGF- $\beta 1$ and PAR-2 in the BALF of fIIPs support the hypothesis regarding interactions of alveolar damage, the coagulation cascade and fibroproliferative healing in fIIPs. We found that PAR-2, TGF- $\beta 1$ and IL-4R $\alpha$ are significantly upregulated in the BALF of fIIPs compared to controls, therefore we suppose that they could become suitable biomarkers of fibrosing interstitial lung diseases (i.e. the markers of fibroproliferative healing), which could be easily detected in BALF using standard ELISA methods. We also observed that the ratios of specific cytokines and receptors could increase the reliability of the method and avoid bias associated with different BALF dilutions.

\section{CONFLICT OF INTEREST}

The authors confirm that this article content has no conflict of interest.

\section{ACKNOWLEDGEMENTS}

The study was supported by grant NS10423-3/2009 and NT 13433-4/2012, IGA MZCR

\section{REFERENCES}

[1] Bargagli E, Prasse A, Olivieri C, Muller-Quernheim J, Rottoli P. Macrophage-derived biomarkers of idiopathic pulmonary fibrosis. Pulm Med 2011; 2011: 717130

[2] Selman M, Pardo A. Idiopathic pulmonary fibrosis: an epithelial/fibroblastic cross-talk disorder. Respir Res 2002; 3: 3 .

[3] Allen JT, Spiteri MA. Growth factors in idiopathic pulmonary fibrosis: relative roles. Respir Res 2002; 3: 13.

[4] Gauldie J, Kolb M, Sime PJ. A new direction in the pathogenesis of idiopathic pulmonary fibrosis? Respir Res 2002; 3: 1 .

[5] Kelly M, Kolb M, Bonniaud P, Gauldie J. Re-evaluation of fibrogenic cytokines in lung fibrosis. Curr Pharm Des 2003; 9: 3949.

[6] Wygrecka M, Kwapiszewska G, Jablonska E, et al. Role of protease-activated receptor-2 in idiopathic pulmonary fibrosis. Am J Respir Crit Care Med 2011; 183(12): 1703-14.

[7] Chambers RC. Procoagulant signalling mechanisms in lung inflammation and fibrosis: novel opportunities for pharmacological intervention? Br J Pharmacol 2008; 153(Suppl 1): S367-78.

[8] Willis BC, Borok Z. TGF-beta-induced EMT: mechanisms and implications for fibrotic lung disease. Am J Physiol Lung Cell Mol Physiol 2007; 293(3): L525-34.

[9] Gorowiec MR, Borthwick LA, Parker SM, Kirby JA, Saretzki GC, Fisher AJ. Free radical generation induces epithelial-tomesenchymal transition in lung epithelium via a TGF- $\beta 1$ dependent mechanism. Free Radic Biol Med 2012; 52(6): 1024-32.
[10] Mackinnon AC, Gibbons MA, Farnworth SL, et al. Regulation of transforming growth factor- $\beta 1$-driven lung fibrosis by galectin-3. Am J Respir Crit Care Med 2012; 185(5): 537-46.

[11] Khalil N, O'Connor RN, Unruh HW, et al. Increased production and immunohistochemical localization of transforming growth factor-beta in idiopathic pulmonary fibrosis. Am J Respir Cell Mol Biol 1991; 5: 155-62.

[12] Armstrong L, Godinho SI, Uppington KM, Whittington HA, Millar $\mathrm{AB}$. Tumour necrosis factor-alpha processing in interstitial lung disease: a potential role for exogenous proteinase-3. Clin Exp Immunol 2009; 156(2): 336-43.

[13] Nhu QM, Shirey KA, Pennini M, Stiltz J, Vogel S. Proteaseactivated receptor 2 activation promotes an anti-inflammatory and alternatively activated phenotype in LPS-stimulated murine macrophages. Innate Immun 2012; 18(2): 193-203.

[14] Pechkovsky DV, Prasse A, Kollert F, et al. Alternatively activated alveolar macrophages in pulmonary fibrosis- mediator production and intracellular signal transduction. Clin Immunol 2010; 137(1): 89-101.

[15] Byers DE, Holtzman MJ. Alternatively activated macrophages as cause or effect in airway disease. Am J Respir Cell Mol Biol 2010; 43(1): 1-4.

[16] Mentink-Kane MM, Cheever AW, Wilson MS, et al. Accelerated and progressive and lethal liver fibrosis in mice that lack interleukin (IL)-10, IL-12p40, and IL-13R $\alpha 2$. Gastroenterology 2011;141(6): 2200-9.

[17] Jakubzick C, Choi ES, Kunkel SL, et al. Augmented pulmonary IL4 and IL-13 receptor subunit expression in idiopathic interstitial pneumonia. J Clin Pathol 2004; 57(5): 477-86.

[18] Vasakova M, Sterclova M, Kolesar L, et al. Cytokine gene polymorphisms and BALF cytokine levels in interstitial lung diseases. Respir Med 2009; 103(5): 773-9.

[19] Vasakova M, Sterclova M, Kolesar L, et al. Bronchoalveolar lavage fluid cellular characteristics, functional parameters and cytokine and chemokine levels in interstitial lung diseases. Scand J Immunol 2009; 69(3): 268-74.

[20] Kinder BW, Brown KK, McCormack FX, et al. Serum surfactant protein-A is a strong predictor of early mortality in idiopathic pulmonary fibrosis. Chest 2009; 135: 1557-63.

[21] Yokoyama A, Kondo K, Nakajima M, et al. Prognostic value of circulating KL-6 in idiopathic pulmonary fibrosis. Respirology 2006; $11: 164-8$.

[22] Borensztajn K, Bresser P, van der Loos C, et al. Protease-activated receptor-2 induces myofibroblast differentiation and tissue factor up-regulation during bleomycin-induced lung injury: potential role in pulmonary fibrosis. Am J Pathol 2010; 177(6): 2753-64.

[23] Fan L, Yotov WV, Zhu T, et al. Tissue factor enhances proteaseactivated receptor-2-mediated factor VIIa cell proliferative properties. J Thromb Haemost 2005; 3(5): 1056-63.

[24] Kasai H, Allen JT, Mason RM, Kamimura T, Zhang Z. TGF-beta1 induces human alveolar epithelial to mesenchymal cell transition (EMT). Respir Res 2005; 6: 56.

[25] $\mathrm{Cu}$ A, Ye Q, Sarria R, Nakamura S, Guzman J, Costabel U. Nacetylcysteine inhibits TNF-alpha, sTNFR, and TGF-beta1 release by alveolar macrophages in idiopathic pulmonary fibrosis in vitro. Sarcoidosis Vasc Diffuse Lung Dis 2009; 26(2): 147-54. 